PROCEEDINGS OF THE

AMERICAN MATHEMATICAL SOCIETY

Volume 138, Number 11, November 2010, Pages 3923-3932

S 0002-9939(2010)10552-X

Article electronically published on June 22, 2010

\title{
A NEW CROSS THEOREM FOR SEPARATELY HOLOMORPHIC FUNCTIONS
}

\author{
MAREK JARNICKI AND PETER PFLUG
}

(Communicated by Franc Forstneric)

\begin{abstract}
We prove a new cross theorem for separately holomorphic func-
\end{abstract} tions.

\section{Introduction. Main RESUlt}

Throughout the paper we will work in the following geometric context; details may be found in [5].

We fix an integer $N \geq 2$ and let $D_{j}$ be a (connected) Riemann domain over $\mathbb{C}^{n_{j}}$, $j=1, \ldots, N$. Let $\varnothing \neq A_{j} \subset D_{j}$ be locally pluriregular, $j=1, \ldots, N$.

We will use the following conventions: $A_{j}^{\prime}:=A_{1} \times \cdots \times A_{j-1}, j=2, \ldots, N$, $A_{j}^{\prime \prime}:=A_{j+1} \times \cdots \times A_{N}, j=1, \ldots, N-1$. Analogously, a point $a=\left(a_{1}, \ldots, a_{N}\right) \in$ $D_{1} \times \cdots \times D_{N}$ may be written as $a=\left(a_{j}^{\prime}, a_{j}, a_{j}^{\prime \prime}\right)$, where $a_{j}^{\prime}:=\left(a_{1}, \ldots, a_{j-1}\right)$, $a_{j}^{\prime \prime}:=\left(a_{j+1}, \ldots, a_{N}\right)$ (with obvious exceptions for $j \in\{1, N\}$ ).

We define an $N$-fold cross

$$
\boldsymbol{X}=\boldsymbol{X}\left(\left(D_{j}, A_{j}\right)_{j=1}^{N}\right):=\bigcup_{j=1}^{N} A_{j}^{\prime} \times D_{j} \times A_{j}^{\prime \prime} .
$$

One may easily prove that $\boldsymbol{X}$ is connected.

We say that a function $f: \boldsymbol{X} \longrightarrow \mathbb{C}$ is separately holomorphic on $\boldsymbol{X}$ (we write $\left.f \in \mathcal{O}_{s}(\boldsymbol{X})\right)$ if for any $j \in\{1, \ldots, N\}$ and $\left(a_{j}^{\prime}, a_{j}^{\prime \prime}\right) \in A_{j}^{\prime} \times A_{j}^{\prime \prime}$, the function $D_{j} \ni$ $z_{j} \longmapsto f\left(a_{j}^{\prime}, z_{j}, a_{j}^{\prime \prime}\right) \in \mathbb{C}$ is holomorphic in $D_{j}$.

Let $h_{A_{j}, D_{j}}$ denote the relative extremal function of $A_{j}$ in $D_{j}, j=1, \ldots, N$. Recall that $h_{A, D}:=\sup \left\{u \in \mathcal{P S H}(D): u \leq 1,\left.u\right|_{A} \leq 0\right\}, A \subset D$ (cf. [6], § 4.5). Put

$$
\widehat{\boldsymbol{X}}:=\left\{\left(z_{1}, \ldots, z_{N}\right) \in D_{1} \times \cdots \times D_{N}: h_{A_{1}, D_{1}}^{*}\left(z_{1}\right)+\cdots+h_{A_{N}, D_{N}}^{*}\left(z_{N}\right)<1\right\},
$$

where ${ }^{*}$ stands for the upper semicontinuous regularization. One may prove that $\widehat{\boldsymbol{X}}$ is connected and $\boldsymbol{X} \subset \widehat{\boldsymbol{X}}$.

The classical cross theorem is the following result:

Received by the editors November 27, 2009.

2010 Mathematics Subject Classification. Primary 32D15.

Key words and phrases. Separately holomorphic function, cross theorem.

This research was partially supported by grant no. N N201 361436 of the Polish Ministry of Science and Higher Education and DFG-grant 436POL113/103/0-2.

(C)2010 American Mathematical Society Reverts to public domain 28 years from publication 
Theorem 1 (12, [13, 15, [14, [8, [9, 10, [7, 1], 16]). For each $f \in \mathcal{O}_{s}(\boldsymbol{X})$ there exists exactly one $\widehat{f} \in \mathcal{O}(\widehat{\boldsymbol{X}})$ such that $\widehat{f}=f$ on $\boldsymbol{X}$ and $\sup _{\widehat{\boldsymbol{X}}}|\widehat{f}|=\sup _{\boldsymbol{X}}|f| \leq$ $+\infty$.

The aim of this paper is to extend the above theorem to a class of more general objects, namely $(N, k)$-crosses $\boldsymbol{X}_{N, k}$ defined for $k \in\{1, \ldots, N\}$ as follows:

$$
\boldsymbol{X}_{N, k}=\mathbb{X}_{N, k}\left(\left(A_{j}, D_{j}\right)_{j=1}^{N}\right):=\bigcup_{\substack{\alpha_{1}, \ldots, \alpha_{N} \in\{0,1\} \\ \alpha_{1}+\cdots+\alpha_{N}=k}} \boldsymbol{x}_{\alpha},
$$

where

$$
x_{\alpha}:=x_{1, \alpha_{1}} \times \cdots \times x_{N, \alpha_{N}}, \quad x_{j, \alpha_{j}}:= \begin{cases}D_{j}, & \text { if } \alpha_{j}=1, \\ A_{j}, & \text { if } \alpha_{j}=0 .\end{cases}
$$

Notice that $N$-fold crosses are just $(N, 1)$-crosses in the above terminology. Obviously, $\boldsymbol{X}_{N, N}=D_{1} \times \cdots \times D_{N}$. Thus, if $N=2$, then in fact we have only $\boldsymbol{X}_{2,1}$.

Recall that the theory of extension of separately holomorphic functions had been first developed for $N=2$. Then the $N$-fold case (obtained via induction) was considered as a natural generalization of $\boldsymbol{X}_{2,1}$. In our opinion, each of the crosses $\boldsymbol{X}_{N, k}$ may be considered as a natural generalization of $\boldsymbol{X}_{2,1}$. Consequently, one should try to find an analogous version of the cross theorem for all $(N, k)$-crosses.

We say that a function $f: \boldsymbol{X}_{N, k} \longrightarrow \mathbb{C}$ is separately holomorphic $\left(f \in \mathcal{O}_{s}\left(\boldsymbol{X}_{N, k}\right)\right)$ if for all $a=\left(a_{1}, \ldots, a_{N}\right) \in A_{1} \times \cdots \times A_{N}$ and $\alpha=\left(\alpha_{1}, \ldots, \alpha_{N}\right) \in\{0,1\}^{N}$ with $|\alpha|=k$, the function

$$
D^{\alpha}:=\prod_{\substack{j \in\{1, \ldots, N\}: \\ \alpha_{j}=1}} D_{j} \ni z \longmapsto f\left(i_{a, \alpha}(z)\right)
$$

is holomorphic, where $i_{a, \alpha}: D^{\alpha} \longrightarrow \boldsymbol{x}_{\alpha}$,

$$
i_{a, \alpha}(z):=\left(w_{1}, \ldots, w_{N}\right), \quad w_{j}:= \begin{cases}z_{j}, & \text { if } \alpha_{j}=1 \\ a_{j}, & \text { if } \alpha_{j}=0 .\end{cases}
$$

Put

$$
\begin{aligned}
& \widehat{\boldsymbol{X}}_{N, k}=\widehat{\mathbb{X}}_{N, k}\left(\left(A_{j}, D_{j}\right)_{j=1}^{N}\right) \\
&:=\left\{\left(z_{1}, \ldots, z_{N}\right) \in D_{1} \times \cdots \times D_{N}: \sum_{j=1}^{N} h_{A_{j}, D_{j}}^{*}\left(z_{j}\right)<k\right\} .
\end{aligned}
$$

Note that $\widehat{\boldsymbol{X}}_{N, N}=D_{1} \times \cdots \times D_{N}$.

Let $\varphi_{j}: D_{j} \longrightarrow \widetilde{D}_{j}$ be the envelope of holomorphy (cf. [4], Definition 1.8.1). Observe that since $\varphi_{j}$ is locally biholomorphic, the set $\widetilde{A}_{j}:=\varphi_{j}\left(A_{j}\right) \subset \widetilde{D}_{j}$ is locally pluriregular, $j=1, \ldots, N$. Let

$$
\widetilde{\boldsymbol{X}}_{N, k}:=\mathbb{X}_{N, k}\left(\left(\widetilde{A}_{j}, \widetilde{D}_{j}\right)_{j=1}^{N}\right), \quad \widehat{\widetilde{\boldsymbol{X}}}_{N, k}:=\widehat{\mathbb{X}}_{N, k}\left(\left(\widetilde{A}_{j}, \widetilde{D}_{j}\right)_{j=1}^{N}\right)
$$

Put

$$
\varphi: D_{1} \times \cdots \times D_{N} \longrightarrow \widetilde{D}_{1} \times \cdots \times \widetilde{D}_{N}, \quad \varphi\left(z_{1}, \ldots, z_{N}\right):=\left(\varphi_{1}\left(z_{1}\right), \ldots, \varphi_{N}\left(z_{N}\right)\right) .
$$

Note that:

- $\varphi\left(\boldsymbol{X}_{N, k}\right) \subset \widetilde{\boldsymbol{X}}_{N, k}$, 
- $\varphi\left(\widehat{\boldsymbol{X}}_{N, k}\right) \subset \widehat{\widetilde{\boldsymbol{X}}}_{N, k}$ (because $\left.h_{\widetilde{A}_{j}, \widetilde{D}_{j}}^{*} \circ \varphi_{j} \leq h_{A_{j}, D_{j}}^{*}, j=1, \ldots, N\right)$.

Our main result is the following cross theorem for $(N, k)$-crosses.

Theorem 2. For every $f \in \mathcal{O}_{s}\left(\boldsymbol{X}_{N, k}\right)$ there exists exactly one $\widehat{f} \in \mathcal{O}\left(\widehat{\widetilde{\boldsymbol{X}}}_{N, k}\right)$ such that $\widehat{f} \circ \varphi=f$ on $\boldsymbol{X}_{N, k}$ and $\sup _{\widehat{\boldsymbol{X}}_{N, k}}|\widehat{f}|=\sup _{\boldsymbol{X}_{N, k}}|f|$.

The proof will be presented in $\$ 5$ and will be based on Theorem 1 and the following lemmas (which might also be useful in other applications).

Lemma 3. Let $G$ be a Riemann domain over $\mathbb{C}^{n}$, let $D \Subset G$ be a Riemann domain of holomorphy, and let $A \subset D$ be non-pluripolar. Put

$$
\Delta(\mu):=\left\{z \in D: h_{A, D}^{*}(z)<\mu\right\}, \quad 0<\mu \leq 1 .
$$

Then

$$
h_{\Delta(r), \Delta(s)}^{*}=\max \left\{0, \frac{h_{A, D}^{*}-r}{s-r}\right\} \text { on } \Delta(s), \quad 0<r<s \leq 1 .
$$

Lemma 4. Assume additionally that $D_{1}, \ldots, D_{N}$ are Riemann domains of holomorphy. Then

$$
\begin{aligned}
& h_{\widehat{\boldsymbol{X}}_{N, k-1}, \widehat{\boldsymbol{X}}_{N, k}}^{*}(z)=\max \left\{0, \sum_{j=1}^{N} h_{A_{j}, D_{j}}^{*}\left(z_{j}\right)-k+1\right\}, \\
& z=\left(z_{1}, \ldots, z_{N}\right) \in \widehat{\boldsymbol{X}}_{N, k}, \quad k \in\{2, \ldots, N\} .
\end{aligned}
$$

To our knowledge the formula from Lemma 3 seems to be new. We do not know whether Lemmas 3, 4are true for arbitrary Riemann domains.

$$
\text { 2. Basic PRoperties of }(N, k) \text {-CROsSes }
$$

Remark 5. (a) $A_{1} \times \cdots \times A_{N} \subset \boldsymbol{X}_{N, k} \subset \widehat{\boldsymbol{X}}_{N, k}$.

(b) $\boldsymbol{X}_{N, k-1} \subset \boldsymbol{X}_{N, k}, \widehat{\boldsymbol{X}}_{N, k-1} \subset \widehat{\boldsymbol{X}}_{N, k}, k=2, \ldots, N$.

(c) $\boldsymbol{X}_{N, k}=\left(\boldsymbol{X}_{N-1, k-1} \times D_{N}\right) \cup\left(\boldsymbol{X}_{N-1, k} \times A_{N}\right), k=2, \ldots, N-1, N \geq 3$.

(d) $\boldsymbol{X}_{N, k}$ and $\widehat{\boldsymbol{X}}_{N, k}$ are connected.

(e) If $\left(D_{j, k}\right)_{k=1}^{\infty}$ is a sequence of subdomains of $D_{j}$ such that $D_{j, k} \nearrow D_{j}$, $D_{j, k} \supset A_{j, k} \nearrow A_{j}, j=1, \ldots, N$, then $\mathbb{X}_{N, k}\left(\left(A_{j, k}, D_{j, k}\right)_{j=1}^{N}\right) \nearrow \boldsymbol{X}_{N, k}$ and

$$
\widehat{\mathbb{X}}_{N, k}\left(\left(A_{j, k}, D_{j, k}\right)_{j=1}^{N}\right) \nearrow \widehat{\boldsymbol{X}}_{N, k} .
$$

(f) If $D_{1}, \ldots, D_{N}$ are domains of holomorphy, then $\widehat{\boldsymbol{X}}_{N, k}$ is a domain of holomorphy.

\section{Proof of Lemma 3}

Let

$$
L:=h_{\Delta(r), \Delta(s)}^{*}, \quad R:=\max \left\{0, \frac{h_{A, D}^{*}-r}{s-r}\right\} .
$$

Put $\Delta[r]:=\left\{z \in D: h_{A, D}^{*}(z) \leq r\right\}$. It is clear that

$$
\begin{gathered}
L=h_{\Delta(r), \Delta(s)} \geq h_{\Delta[r], \Delta(s)}^{*} \geq h_{\Delta[r], \Delta(s)} \geq R, \\
L=R=0 \text { on } \Delta(r), \quad R=0 \text { on } \Delta[r] .
\end{gathered}
$$

Step 1. Reduction to the case $s=1$. 
Suppose that $0<r<s<1$. Observe that $\Delta(s)$ is a Riemann region of holomorphy. Moreover, $h_{A \cap \Delta(s), \Delta(s)}^{*}=(1 / s) h_{A, D}^{*}$ on $\Delta(s)$.

Indeed, it obvious that $h_{A \cap \Delta(s), \Delta(s)}^{*} \geq(1 / s) h_{A, D}^{*}$ on $\Delta(s)$. Let $u \in \mathcal{P} \mathcal{S H}(\Delta(s))$, $u \leq 1, u \leq 0$ on $A \cap \Delta(s)$. Observe that for every $z_{0} \in D \cap \partial(\Delta(s))$ we have $\limsup _{z \rightarrow z_{0}} u(z) \leq 1 \leq(1 / s) h_{A, D}^{*}\left(z_{0}\right)$. Thus, the function

$$
v:= \begin{cases}\max \left\{s u, h_{A, D}^{*}\right\} & \text { on } \Delta(s) \\ h_{A, D}^{*} & \text { on } D \backslash \Delta(s)\end{cases}
$$

is plurisubharmonic on $D$. It is known that there exists a pluripolar set $P \subset A$ such that $h_{A, D}^{*}=0$ on $A \backslash P$ (cf. [6]). Hence, $A \backslash P \subset \Delta(s), v \leq h_{A \backslash P, D}^{*}=h_{A, D}^{*}$, and therefore, $h_{A \cap \Delta(s), \Delta(s)}^{*} \leq(1 / s) h_{A, D}^{*}$ on $\Delta(s)$.

In particular, $A \cap S$ is not pluripolar for every connected component $S$ of $\Delta(s)$. Hence,

$$
L=h_{\Delta(r), \Delta(s)}=h_{\left\{h_{A, \Delta(s)}^{*}<r / s\right\}, \Delta(s)}, \quad R=\max \left\{0, \frac{h_{A, \Delta(s)}^{*}-r / s}{1-r / s}\right\} .
$$

Thus the problem for $(D, A, r, s)$ reduces to $(S, A \cap S, r / s, 1)$, where $S$ is a connected component of $\Delta(s)$.

From now on we assume that $s=1$.

Step 2. Approximation. Let $A_{\nu} \nearrow A, D_{\nu} \nearrow D$, where $A_{\nu} \subset D_{\nu}$ is nonpluripolar, $\nu \in \mathbb{N}$. Suppose that the formula holds for each $\left(D_{\nu}, A_{\nu}, r\right)$. Then it holds for $(D, A, r)$.

Indeed, we know that $h_{A_{\nu}, D_{\nu}}^{*} \searrow h_{A, D}^{*}$. Hence $\left\{h_{A_{\nu}, D}^{*}<r\right\} \nearrow \Delta(r)$. Thus $h_{\left\{h_{A_{\nu}, D}^{*}<r\right\}, D}^{*} \searrow h_{\Delta(r), D}^{*}$

Step 3. The case where $D$ is hyperconvex, $A$ is compact, and $h_{A, D}^{*}$ is continuous.

Let $u \in \mathcal{P S H}(D), u \leq 1, u \leq 0$ on $\Delta[r]$. Using continuity of $h_{A, D}^{*}$ and [6], Proposition 4.5.2, we easily conclude that $\Delta[r]$ is compact. Let $U:=D \backslash \Delta[r]$. Observe that for $z_{0} \in \partial U$ we get

$$
\liminf _{U \ni z \rightarrow z_{0}}\left(h_{A, D}^{*}(z)-(1-r) u(z)-r\right) \geq 0 .
$$

Hence, by the domination principle (cf. [6], Corollary 3.7.4), $(1-r) u+r \leq h_{A, D}^{*}$ in $U$. This shows that $h_{\Delta[r], D} \leq R$. Thus, by $\left(^{*}\right)$, we get $h_{\Delta[r], D}^{*} \equiv R$ for all $0<r<1$. Observe that $\Delta\left[r_{\nu}\right] \nearrow \Delta(r)$ for $0<r_{\nu} \nearrow r$. Consequently, $L \equiv R$.

Step 4. The case where $D$ is hyperconvex and $A$ is compact.

Let $A^{(\varepsilon)}:=\bigcup_{a \in A} \widehat{\widehat{\mathbb{P}}(a, \varepsilon)}$, where $\widehat{\mathbb{P}}(a, \varepsilon)$ stands for the "polydisc" in the sense of the Riemann domain $D\left(A^{(\varepsilon)}\right.$ is defined for small $\left.\varepsilon>0\right)$. By [6], Corollary 4.5.9, we know that $h_{A^{(\varepsilon)}, D}=h_{A^{(\varepsilon)}, D}^{*}$ is continuous. Thus, using Step 3 and $\left(^{*}\right)$, we get

$$
h_{\left\{h_{A^{(\varepsilon)}, D} \leq r\right\}, D}=\max \left\{0, \frac{h_{A^{(\varepsilon)}, D}-r}{1-r}\right\}, \quad 0<\varepsilon \ll 1 .
$$

By [6], Proposition 4.5.10, we have $h_{A^{(\varepsilon)}, D} \nearrow h_{A, D}$ as $\varepsilon \searrow 0$. In particular,

$$
\left\{h_{A^{(\varepsilon)}, D} \leq r\right\} \searrow\left\{h_{A, D} \leq r\right\} \text { as } \varepsilon \searrow 0 \text {. }
$$

Hence, once again by [6], Proposition 4.5.10,

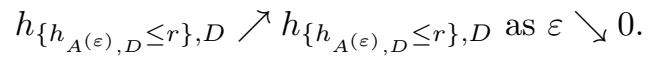


Consequently,

$$
h_{\left\{h_{A, D} \leq r\right\}, D}=\max \left\{0, \frac{h_{A, D}-r}{1-r}\right\} \leq R .
$$

Thus $h_{\left\{h_{A, D} \leq r\right\}, D}^{*} \leq R$. Observe that the set $\left\{h_{A, D} \leq r\right\} \backslash \Delta[r]$ is pluripolar. Consequently, $h_{\Delta[r], D}^{*} \leq R$. We finish the proof as in Step 3 .

Step 5. The case where $A$ is open.

We use Step 4 and approximation (Step 2) with $A_{\nu} \nearrow A, D_{\nu} \nearrow D$, where $A_{\nu} \Subset D_{\nu}$ is compact non-pluripolar and $D_{\nu}$ is hyperconvex, $\nu \in \mathbb{N}$.

Step 6. The case where $D$ is hyperconvex and $A \Subset D$ is non-pluripolar.

By Step 5 we have

$$
h_{\left\{h_{\Delta(\varepsilon), D}^{*}<r\right\}, D}^{*}=\max \left\{0, \frac{h_{\Delta(\varepsilon), D}^{*}-r}{1-r}\right\}, \quad 0<\varepsilon<1 .
$$

By [2] we get

$$
\frac{h_{A, D}^{*}-\varepsilon}{1-\varepsilon} \leq h_{\Delta(\varepsilon), D}^{*} \leq h_{A, D}^{*}
$$

in particular, $h_{\Delta(\varepsilon), D}^{*} \nearrow h_{A, D}^{*}$ as $\varepsilon \searrow 0$. Moreover,

$$
\left\{h_{\Delta(\varepsilon), D}^{*}<\frac{r-\varepsilon}{1-\varepsilon}\right\} \subset \Delta(r) \subset\left\{h_{\Delta(\varepsilon), D}^{*}<r\right\}, \quad 0<\varepsilon<r .
$$

Consequently,

$$
\begin{aligned}
\max \left\{0, \frac{h_{\Delta(\varepsilon), D}^{*}-\frac{r-\varepsilon}{1-\varepsilon}}{1-\frac{r-\varepsilon}{1-\varepsilon}}\right\} & =h_{\left\{h_{\Delta(\varepsilon), D}^{*}<\frac{r-\varepsilon}{1-\varepsilon}\right\}, D}^{*} \\
& \geq h_{\Delta(r), D}^{*} \geq h_{\left\{h_{\Delta(\varepsilon), D}^{*}<r\right\}, D}^{*}=\max \left\{0, \frac{h_{\Delta(\varepsilon), D}^{*}-r}{1-r}\right\}, \quad 0<\varepsilon<r .
\end{aligned}
$$

Letting $\varepsilon \searrow 0$, we get the required formula.

Step 7. The general case.

We use Step 6 and approximation (Step 2) with $A_{\nu} \nearrow A, D_{\nu} \nearrow D$, where $A_{\nu} \Subset D_{\nu}$ is non-pluripolar and $D_{\nu}$ is hyperconvex, $\nu \in \mathbb{N}$.

The proof of Lemma 3 is completed.

\section{Proof of Lemma 4}

By Remark 5 (e), we may assume that $A_{j} \Subset D_{j} \Subset G_{j}$, where $G_{j}$ is a Riemann domain over $\mathbb{C}^{n_{j}}, j=1, \ldots, N$. Fix $2 \leq k \leq N$. Let

$$
h_{j}:=h_{A_{j}, D_{j}}^{*}, j=1, \ldots, N, \quad h\left(z_{1}, \ldots, z_{N}\right):=h_{1}\left(z_{1}\right)+\cdots+h_{N}\left(z_{N}\right) .
$$

Let

$$
\begin{array}{r}
L_{N, k}:=h_{\widehat{\boldsymbol{X}}_{N, k-1}, \widehat{\boldsymbol{X}}_{N, k}}^{*}, \quad R_{N, k}(z)=\max \left\{0, \sum_{j=1}^{N} h_{A_{j}, D_{j}}^{*}\left(z_{j}\right)-k+1\right\}, \\
z=\left(z_{1}, \ldots, z_{N}\right) \in \widehat{\boldsymbol{X}}_{N, k} .
\end{array}
$$

It is clear that $L_{N, k} \geq R_{N, k}$ and $L_{N, k}=R_{N, k}=0$ on $\widehat{\boldsymbol{X}}_{N, k-1}$. Fix an $a=$ $\left(a_{1}, \ldots, a_{N}\right) \in \widehat{\boldsymbol{X}}_{N, k} \backslash \widehat{\boldsymbol{X}}_{N, k-1}$. We may assume that $h_{1}\left(a_{1}\right) \leq \cdots \leq h_{N}\left(a_{N}\right)$. Suppose that $h_{1}\left(a_{1}\right)=\cdots=h_{s}\left(a_{s}\right)=0$ and $h_{s+1}\left(a_{s+1}\right), \ldots, h_{N}\left(a_{N}\right)>0$ for an 
$s \in\{0, \ldots, N\}$. Since $h(a) \geq k-1$, we see that in fact $s \leq N-k \leq N-2$. In particular, if $N=2$, then $s=0$.

Let $\widehat{\boldsymbol{Y}}_{N-s, p}=\widehat{\mathbb{X}}_{N-s, p}\left(\left(A_{j}, D_{j}\right)_{j=s+1}^{N}\right), p \in\{k-1, k\}$. Observe that

$$
\left\{a_{1}, \ldots, a_{s}\right\} \times \widehat{\boldsymbol{Y}}_{N-s, p} \subset \widehat{\boldsymbol{X}}_{N, p}, \quad p \in\{k-1, k\} .
$$

Consequently,

$$
h_{\widehat{\boldsymbol{X}}_{N, k-1}, \widehat{\boldsymbol{X}}_{N, k}}^{*}(a) \leq h_{\widehat{\boldsymbol{Y}}_{N-s, k-1}, \widehat{\boldsymbol{Y}}_{N-s, k}}^{*}\left(a_{s+1}, \ldots, a_{N}\right) .
$$

Thus, if we know that $L_{N-s, k}\left(a_{s+1}, \ldots, a_{N}\right) \leq R_{N-s, k}\left(a_{s+1}, \ldots, a_{N}\right)$, then

$$
L_{N, k}(a) \leq R_{N-s, k}\left(a_{s+1}, \ldots, a_{N}\right)=R_{N, k}(a) .
$$

This reduces the proof to the case $s=0$, i.e. $h_{j}\left(a_{j}\right)>0, j=1, \ldots, N$.

Put

$$
\Delta_{j, t}:=\left\{z_{j} \in D_{j}: h_{j}\left(z_{j}\right)<t\right\}, \quad j=1, \ldots, N .
$$

Take $0<r_{j}<s_{j} \leq 1, j=1, \ldots, N$, such that $r_{1}+\cdots+r_{N}=k-1$ and $s_{1}+\cdots+s_{N}=k$. Observe that

$$
\Delta_{1, r_{1}} \times \cdots \times \Delta_{N, r_{N}} \subset \widehat{\boldsymbol{X}}_{N, k-1}, \quad \Delta_{1, s_{1}} \times \cdots \times \Delta_{N, s_{N}} \subset \widehat{\boldsymbol{X}}_{N, k} .
$$

Hence, using the product property for the relative extremal function (cf. [3], Theorem 4.1) and Lemma 3, we get

$$
\begin{aligned}
& L_{N, k}(z) \leq h_{\Delta_{1, r_{1}} \times \cdots \times \Delta_{N, r_{N}}, \Delta_{1, s_{1}} \times \cdots \times \Delta_{N, s_{N}}}^{*}(z) \\
&=\max \left\{h_{\Delta_{1, r_{1}}, \Delta_{1, r_{1}}}^{*}\left(z_{1}\right), \ldots, h_{\Delta_{N, r_{N}}, \Delta_{N, r_{N}}}^{*}\left(z_{N}\right)\right\} \\
&=\max \left\{0, \frac{h_{1}\left(z_{1}\right)-r_{1}}{s_{1}-r_{1}}, \ldots, \frac{h_{N}\left(z_{N}\right)-r_{N}}{s_{N}-r_{N}}\right\}, \\
& z=\left(z_{1}, \ldots, z_{N}\right) \in \Delta_{1, s_{1}} \times \cdots \times \Delta_{N, s_{N}} .
\end{aligned}
$$

Observe that there exist numbers $s_{1}, \ldots, s_{N} \in(0,1]$ such that $s_{1}+\cdots+s_{N}=k$ and

$$
h_{j}\left(a_{j}\right)<s_{j}<\frac{h_{j}\left(a_{j}\right)}{h(a)-k+1}, \quad j=1, \ldots, N .
$$

Indeed, since the case where $h(a)=k-1$ is trivial, we may assume that $h(a)>$ $k-1$. Note that $h_{j}\left(a_{j}\right)<\frac{h_{j}\left(a_{j}\right)}{h(a)-k+1}, j=1, \ldots, N$. Suppose that

$$
\frac{h_{j}\left(a_{j}\right)}{h(a)-k+1} \leq 1, j=1, \ldots, \sigma, \quad \frac{h_{j}\left(a_{j}\right)}{h(a)-k+1}>1, j=\sigma+1, \ldots, N,
$$

for a $\sigma \in\{0, \ldots, N\}$. Observe that

$$
\sum_{j=1}^{N} \frac{h_{j}\left(a_{j}\right)}{h(a)-k+1}=\frac{h(a)}{h(a)-k+1}>k,
$$

so the case $\sigma=N$ is simple. Thus, assume that $\sigma \leq N-1$. We only need to show that

$$
\left(\sum_{j=1}^{\sigma} \frac{h_{j}\left(a_{j}\right)}{h(a)-k+1}\right)+N-\sigma>k .
$$


The case where $\sigma \leq N-k$ is obvious. Thus assume that $\sigma \geq N-k+1$. We have to show that

$$
\begin{aligned}
& \sum_{j=1}^{\sigma} h_{j}\left(a_{j}\right)>(h(a)-k+1)(k-N+\sigma) \\
= & (k-1-N+\sigma) h(a)+\left(\sum_{j=1}^{\sigma} h_{j}\left(a_{j}\right)\right)+\left(\sum_{j=\sigma+1}^{N} h_{j}\left(a_{j}\right)\right)+(-k+1)(k-N+\sigma)
\end{aligned}
$$

or, equivalently,

$$
(k-1-N+\sigma) h(a)+\left(\sum_{j=\sigma+1}^{N} h_{j}\left(a_{j}\right)\right)<(k-1)(k-N+\sigma) .
$$

We have

$$
\begin{aligned}
(k-1-N+\sigma) h(a)+( & \left.\sum_{j=\sigma+1}^{N} h_{j}\left(a_{j}\right)\right) \\
& <(k-1-N+\sigma) k+N-\sigma \leq(k-1)(k-N+\sigma),
\end{aligned}
$$

which gives the required inequality.

Now, define

$$
r_{j}:=\frac{h_{j}\left(a_{j}\right)-s_{j}(h(a)-k+1)}{k-h(a)}, \quad j=1, \ldots, N .
$$

Then:

- $r_{j}>0$ because $s_{j}<\frac{h_{j}\left(a_{j}\right)}{h(a)-k+1}$,

- $r_{j}<s_{j}$ because $h_{j}(a)<s_{j}$,

- $r_{1}+\cdots+r_{N}=k-1$,

- $\frac{h_{j}\left(a_{j}\right)-r_{j}}{s_{j}-r_{j}}=h(a)-k+1, j=1, \ldots, N$.

Thus

$$
\begin{aligned}
& L_{N, k}(a) \leq \max \left\{0, \frac{h_{1}\left(a_{1}\right)-r_{1}}{s_{1}-r_{1}}, \ldots, \frac{h_{N}\left(a_{N}\right)-r_{N}}{s_{N}-r_{N}}\right\} \\
& \quad=\max \{0, h(a)-k+1\}=R_{N, k}(a) .
\end{aligned}
$$

The proof of Lemma 4 is completed.

\section{Proof of Theorem 2}

First we prove that for each function $f \in \mathcal{O}_{s}\left(\boldsymbol{X}_{N, k}\right)$ there exists exactly one $\tilde{f} \in \mathcal{O}_{s}\left(\widetilde{\boldsymbol{X}}_{N, k}\right)$ such that $\tilde{f} \circ \varphi \equiv f$ and $\sup _{\widetilde{\boldsymbol{X}}_{N, k}}|\widetilde{f}|=\sup _{\boldsymbol{X}_{N, k}}|f|$.

Indeed, fix an $f \in \mathcal{O}_{s}\left(\boldsymbol{X}_{N, k}\right)$. Take $a=\left(a_{1}, \ldots, a_{N}\right), b=\left(b_{1}, \ldots, b_{N}\right) \in A_{1} \times$ $\cdots \times A_{N}$ and $\alpha=\left(\alpha_{1}, \ldots, \alpha_{N}\right), \beta=\left(\beta_{1}, \ldots, \beta_{N}\right) \in\{0,1\}^{N}$ with $|\alpha|=|\beta|=k$. To simplify notation, suppose that $\alpha=(1, \ldots, 1,0, \ldots, 0)$.

Observe that if $\varphi_{j}\left(a_{j}\right)=\varphi_{j}\left(b_{j}\right), j=k+1, \ldots, N$, then $f\left(\cdot, a_{k+1}, \ldots, a_{N}\right) \equiv$ $f\left(\cdot, b_{k+1}, \ldots, b_{N}\right)$ on $D_{1} \times \cdots \times D_{k}$.

Indeed, since $\varphi_{j}: D_{j} \longrightarrow \widetilde{D}_{j}$ is the envelope of holomorphy, for each $g_{j} \in \mathcal{O}\left(D_{j}\right)$, there exists a $\widetilde{g}_{j} \in \mathcal{O}\left(\widetilde{D}_{j}\right)$ such that $g_{j} \equiv \widetilde{g}_{j} \circ \varphi_{j}$. In particular, if $\varphi\left(z_{j}\right)=\varphi\left(w_{j}\right)$, 
then $g_{j}\left(z_{j}\right)=g_{j}\left(w_{j}\right)$. Take arbitrary $c_{j} \in A_{j}, j=1, \ldots, k$. Then

$$
\begin{aligned}
f\left(c_{1}, \ldots, c_{k}, a_{k+1}, \ldots, a_{N}\right)=f\left(c_{1}, \ldots, c_{k}, b_{k+1}, a_{k+2}, \ldots, a_{N}\right) \\
=\cdots=f\left(c_{1}, \ldots, c_{k}, b_{k+1}, \ldots, b_{N}\right) .
\end{aligned}
$$

Thus $f\left(\cdot, a_{k+1}, \ldots, a_{N}\right)=f\left(\cdot, b_{k+1}, \ldots, b_{N}\right)$ on $A_{1} \times \cdots \times A_{k}$. It remains to use the identity principle.

Recall that

$$
\left(\varphi_{1} \times \cdots \times \varphi_{k}\right): D_{1} \times \cdots \times D_{k} \longrightarrow \widetilde{D}_{1} \times \cdots \times \widetilde{D}_{k}
$$

is the envelope of holomorphy (cf. 4, Proposition 1.8.15 (b)). Consequently, the function

$$
\widetilde{f}_{\alpha}\left(\cdot, \varphi_{k+1}\left(a_{k+1}\right), \ldots, \varphi_{N}\left(a_{N}\right)\right):=\left(\left(\varphi_{1} \times \cdots \times \varphi_{k}\right)^{*}\right)^{-1}\left(f\left(\cdot, a_{k+1}, \ldots, a_{N}\right)\right)
$$

is well defined on

$$
\widetilde{x}_{\alpha}:=\widetilde{D}_{1} \times \cdots \times \widetilde{D}_{k} \times \widetilde{A}_{k+1} \times \cdots \times \widetilde{A}_{N}
$$

with $\tilde{f}_{\alpha} \circ \varphi=f$ on $\boldsymbol{X}_{\alpha}$ and $\sup _{\tilde{\boldsymbol{x}}_{\alpha}}\left|\tilde{f}_{\alpha}\right|=\sup _{\boldsymbol{x}_{\alpha}}|f|$.

In particular, $\widetilde{f}_{\alpha} \circ \varphi=f=\widetilde{f}_{\beta} \circ \varphi$ on $A_{1} \times \cdots \times A_{N}$. Hence, by the identity principle, $\tilde{f}_{\alpha}=\widetilde{f}_{\beta}$ on $\widetilde{\boldsymbol{x}}_{\alpha} \cap \widetilde{\boldsymbol{x}}_{\beta}$.

Thus, we may replace $\left(\left(D_{j}, A_{j}\right)_{j=1}^{N}, \boldsymbol{X}_{N, k}, \widehat{\widetilde{\boldsymbol{X}}}_{N, k}\right)$ by $\left(\left(\widetilde{D}_{j}, \widetilde{A}_{j}\right)_{j=1}^{N}, \widetilde{\boldsymbol{X}}_{N, k}, \widehat{\widetilde{\boldsymbol{X}}}_{N, k}\right)$. So we may assume that $D_{j}$ is a domain of holomorphy and $\varphi_{j}=$ id, $j=1, \ldots, N$.

Moreover, by Remark 5 (e), we may reduce the problem to the case where $A_{j} \Subset$ $D_{j} \Subset G_{j}$, where $G_{j}$ is a Riemann domain over $\mathbb{C}^{n_{j}}, j=1, \ldots, N$.

The case $k=N$ is trivial. The case $k=1$ is the classical cross theorem (Theorem 11). In particular, there is nothing to prove for $N=2$. We apply induction on $N$. Suppose that the result is true for $N-1 \geq 2$.

Now, we apply finite induction on $k$. The case $k=1$ is known. Suppose that the result is true for $k-1$ with $2 \leq k \leq N-1$.

Fix an $f \in \mathcal{O}_{s}\left(\boldsymbol{X}_{N, k}\right)$ and let $C:=\sup _{\boldsymbol{X}_{N, k}}|f|$. Recall that

$$
\boldsymbol{X}_{N, k}=\left(\boldsymbol{X}_{N-1, k-1} \times D_{N}\right) \cup\left(\boldsymbol{X}_{N-1, k} \times A_{N}\right) .
$$

For each $z_{N} \in D_{N}$ the function $f\left(\cdot, z_{N}\right)$ belongs to $\mathcal{O}_{s}\left(\boldsymbol{X}_{N-1, k-1}\right)$. By the inductive assumption there exists a $g_{z_{N}} \in \mathcal{O}\left(\widehat{\boldsymbol{X}}_{N-1, k-1}\right)$ such that $g_{z_{N}}=f\left(\cdot, z_{N}\right)$ on $\boldsymbol{X}_{N-1, k-1}$ and $\sup _{\widehat{\boldsymbol{X}}_{N-1, k-1}}\left|g_{z_{N}}\right| \leq C$. Analogously, for each $z_{N} \in A_{N}$ there exists an $h_{z_{N}} \in \mathcal{O}\left(\widehat{\boldsymbol{X}}_{N-1, k}\right)$ such that $h_{z_{N}}=f\left(\cdot, z_{N}\right)$ on $\boldsymbol{X}_{N-1, k}$ and $\sup _{\widehat{\boldsymbol{X}}_{N-1, k}}\left|h_{z_{N}}\right| \leq$ $C$. Recall that $\widehat{\boldsymbol{X}}_{N-1, k-1} \subset \widehat{\boldsymbol{X}}_{N-1, k}$ and $A_{1} \times \cdots \times A_{N-1} \subset \boldsymbol{X}_{N-1, k-1} \cap \boldsymbol{X}_{N-1, k}$. Since the set $A_{1} \times \cdots \times A_{N-1}$ is not pluripolar, we get $g_{z_{N}}=h_{z_{N}}$ on $\widehat{\boldsymbol{X}}_{N-1, k-1}$ for $z_{N} \in A_{N}$.

Consider the 2 -fold cross

$$
\boldsymbol{Y}:=\mathbb{X}\left(\widehat{\boldsymbol{X}}_{N-1, k-1}, A_{N} ; \widehat{\boldsymbol{X}}_{N-1, k}, D_{N}\right)=\left(\widehat{\boldsymbol{X}}_{N-1, k-1} \times D_{N}\right) \cup\left(\widehat{\boldsymbol{X}}_{N-1, k} \times A_{N}\right)
$$

and let $F: \boldsymbol{Y} \longrightarrow \mathbb{C}$,

$$
F\left(z^{\prime}, z_{N}\right):= \begin{cases}g_{z_{N}}\left(z^{\prime}\right), & \text { if }\left(z^{\prime}, z_{N}\right) \in \widehat{\boldsymbol{X}}_{N-1, k-1} \times D_{N}, \\ h_{z_{N}}\left(z^{\prime}\right), & \text { if }\left(z^{\prime}, z_{N}\right) \in \widehat{\boldsymbol{X}}_{N-1, k} \times A_{N} .\end{cases}
$$


Obviously, $\sup _{\boldsymbol{Y}}|F| \leq C$. To see that $F \in \mathcal{O}_{s}(\boldsymbol{Y})$, we have to prove that for each $z^{\prime} \in \widehat{\boldsymbol{X}}_{N-1, k-1}$, the function $D_{N} \ni z_{N} \longmapsto F\left(z^{\prime}, z_{N}\right)$ is holomorphic. We know that $F\left(\cdot, z_{N}\right)$ is holomorphic for each $z_{N} \in D_{N}$. Let

$$
\boldsymbol{Z}_{N-1, k-1}:=\mathbb{X}_{N-1, k-1}\left(\left(A_{j}, D_{j}\right)_{j=2}^{N}\right) .
$$

Analogously as above, for each $z_{1} \in D_{1}$ there exists a $\varphi_{z_{1}} \in \mathcal{O}\left(\widehat{\boldsymbol{Z}}_{N-1, k-1}\right)$ such that $\varphi_{z_{1}}=f\left(z_{1}, \cdot\right)$ on $\boldsymbol{Z}_{N-1, k-1}$. Thus

$$
\begin{aligned}
& F\left(z_{1}, \ldots, z_{N}\right)=f\left(z_{1}, \ldots, z_{N}\right)=\varphi_{z_{1}}\left(z_{2}, \ldots, z_{N}\right), \\
& \left(z_{1}, \ldots, z_{N}\right) \in\left(\boldsymbol{X}_{N-1, k-1} \times D_{N}\right) \cap\left(D_{1} \times \boldsymbol{Z}_{N-1, k-1}\right) \supset A_{1} \times \cdots \times A_{N-1} \times D_{N} .
\end{aligned}
$$

Consequently, $F\left(z^{\prime}, \cdot\right) \in \mathcal{O}\left(D_{N}\right)$ for $z^{\prime} \in A_{1} \times \cdots \times A_{N-1}$ and hence, using Terada's theorem (cf. e.g. [11]), we conclude that $F \in \mathcal{O}\left(\widehat{\boldsymbol{X}}_{N-1, k-1} \times D_{N}\right)$.

Now, by the classical cross theorem (Theorem 1) with $N=2$, there exists an $\widehat{f} \in \mathcal{O}(\widehat{\boldsymbol{Y}})$ such that $\widehat{f}=F$ on $\boldsymbol{Y}$ (in particular, $\widehat{f}=f$ on $\boldsymbol{X}_{N, k}$ ) and $\sup _{\widehat{\boldsymbol{Y}}}|\widehat{f}| \leq C$. Recall that

$$
\widehat{\boldsymbol{Y}}=\left\{\left(z^{\prime}, z_{N}\right) \in \widehat{\boldsymbol{X}}_{N-1, k} \times D_{N}: h_{\widehat{\boldsymbol{X}}_{N-1, k-1}, \widehat{\boldsymbol{X}}_{N-1, k}}^{*}\left(z^{\prime}\right)+h_{A_{N}, D_{N}}^{*}\left(z_{N}\right)<1\right\} .
$$

Thus, it remains to apply Lemma 4 .

The proof of Theorem 2 is completed.

\section{REFERENCES}

1. O. Alehyane, A. Zeriahi, Une nouvelle version du théorème d'extension de Hartogs pour les applications séparément holomorphes entre espaces analytiques, Ann. Polon. Math. 76 (2001), 245-278. MR1841529 (2002f:32018)

2. Z. Błocki, Equilibrium measure of a product subset of $\mathbb{C}^{n}$, Proc. Amer. Math. Soc. 128 (2000), 3595-3599. MR.1707508 (2001b:32072)

3. A. Edigarian, Analytic discs method in complex analysis, Diss. Math. 402 (2002), 1-56. MR.1897580 (2002m:32049)

4. M. Jarnicki, P. Pflug, Extension of Holomorphic Functions, de Gruyter Expositions in Mathematics 34, Walter de Gruyter, 2000. MR.1797263 (2001k:32017)

5. M. Jarnicki, P. Pflug, A general cross theorem with singularities, Analysis Munich 27 (2007), 181-212. MR2359932 (2009i:32009)

6. M. Klimek, Pluripotential Theory, The Clarendon Press, Oxford University Press, 1991. MR.1150978 (93h:32021)

7. Nguyen Thanh Van, Separate analyticity and related subjects, Vietnam J. Math. 25 (1997), 81-90. MR:1681531 (99m:32002)

8. Nguyen Thanh Van, J. Siciak, Fonctions plurisousharmoniques extrémales et systèmes doublement orthogonaux de fonctions analytiques, Bull. Sci. Math. 115 (1991), 235-244. MR1101026 (92a:32018)

9. Nguyen Thanh Van, A. Zeriahi, Une extension du théorème de Hartogs sur les fonctions séparément analytiques, in Analyse Complexe Multivariables, Récents Dévelopements, A. Meril (éd.), EditEl, Rende, 1991, 183-194. MR1228880(94e:32004)

10. Nguyen Thanh Van, A. Zeriahi, Systèmes doublement othogonaux de fonctions holomorphes et applications, Banach Center Publ. 31, 1995, 281-297. MR1341397 (96g:32004)

11. P. Pflug, Extension of separately holomorphic functions - a survey 1899-2001, Proc. Conf. Complex Analysis (Bielsko-Biała, 2001), Ann. Polon Math. 80 (2003), 21-36. MR.1972831 (2004k:32014)

12. J. Siciak, Analyticity and separate analyticity of functions defined on lower dimensional subsets of $\mathbb{C}^{n}$, Zeszyty Naukowe UJ 13 (1969), 53-70. MR0247132 (40:401)

13. J. Siciak, Separately analytic functions and envelopes of holomorphy of some lower dimensional subsets of $\mathbb{C}^{n}$, Ann. Polon. Math. 22 (1969-1970), 147-171. MR0252675 (40:5893)

14. J. Siciak, Extremal plurisubharmonic functions in $\mathbb{C}^{n}$, Ann. Polon. Math. 39 (1981), 175-211. MR617459 (83e:32018) 
15. V.P. Zahariuta, Separately analytic functions, generalizations of Hartogs theorem, and envelopes of holomorphy, Math. USSR-Sb. 30 (1976), 51-67.

16. A. Zeriahi, Comportement asymptotique des systèmes doublement orthogonaux de Bergman: une approche élémentaire, Vietnam J. Math. 30 (2002), 177-188. MR1934347 (2003g:32010)

Institute of Mathematics, Jagiellonian University, Łojasiewicza 6, 30-348 Kraków, POLAND

E-mail address: Marek.Jarnicki@im.uj.edu.pl

Institut für Mathematik, Carl von Ossietzky Universität Oldenburg, Postfach 2503, D-26111 Oldenburg, Germany

E-mail address: peter.pflug@uni-oldenburg.de 